\title{
Bronchoplasty and pulmonary arterioplasty
}

\author{
Giulio Maurizi ${ }^{1}$, Camilla Vanni ${ }^{1}$, Antonio D’Andrilli ${ }^{1}$, Anna Maria Ciccone ${ }^{1}$, Mohsen Ibrahim $^{1}$, \\ Claudio Andreetti ${ }^{1}$, Cecilia Menna ${ }^{1}$, Camilla Poggi ${ }^{2}$, Federico Venuta $^{2}$, Erino Angelo Rendina ${ }^{1}$ \\ ${ }^{1}$ Department of Thoracic Surgery, Sant'Andrea Hospital, Sapienza University of Rome, Rome, Italy; ${ }^{2}$ Department of Thoracic Surgery, \\ Policlinico Umberto I, Sapienza University of Rome, Rome, Italy \\ Contributions: (I) Conception and design: G Maurizi, C Vanni, A D’Andrilli; (II) Administrative support: G Maurizi, C Vanni; (III) Provision of study \\ materials or patients: C Vanni, AM Ciccone, M Ibrahim, C Andreetti, C Menna, C Poggi; (IV) Collection and assembly of data: C Vanni, C Poggi, C \\ Menna; (V) Data analysis and interpretation: G Maurizi; (VI) Manuscript writing: All authors; (VII) Final approval of manuscript: All authors. \\ Correspondence to: Giulio Maurizi, MD, PhD. Department of Thoracic Surgery, Sant'Andrea Hospital, Sapienza University of Rome, Via di \\ Grottarossa, 1035, Rome 00189, Italy. Email: giulio.maurizi@uniroma1.it.
}

\begin{abstract}
Current results from the literature support the increasingly widespread use of bronchovascular reconstructive operations for the treatment of centrally located lung tumors, even in functionally normal patients. These surgical procedures actually provide the complete oncologic resection of pneumonectomy (PN) with the functional advantages of standard lobectomy, including when performed after induction chemotherapy. When planning such operations, the thorough preoperative assessment of tumor extent, careful surgical technique, and vigilant perioperative management are mandatory in order to achieve the best possible outcome for the patient with the least cost.
\end{abstract}

Keywords: Sleeve resection; pulmonary artery reconstruction (PA reconstruction); pneumonectomy (PN)

Received: 09 May 2019; Accepted: 21 May 2019; published: 05 June 2019.

doi: $10.21037 /$ ccts.2019.05.01

View this article at: http://dx.doi.org/10.21037/ccts.2019.05.01

\section{Introduction}

In step with the growing surgical skills and experience in managing any perioperative issues, bronchoplasty and pulmonary artery (PA) reconstruction techniques, historically developed to avoid pneumonectomy (PN) in patients with impaired cardiopulmonary function, have gained, in recent years, widespread use. Data from the latest published experiences have shown how these procedures, although challenging with respect to standard major lung resections, can provide overall better outcomes in comparison to $\mathrm{PN}$ when performed by highly experienced thoracic surgeons (1-4).

Promising clinical and oncological results have gradually led to the preferred use of lung sparing plastic procedures in the treatment of most centrally located lung tumors, even in patients with good functional reserve. This strategy, in turn, has encouraged the development of new techniques which have further improved the reliability of bronchovascular reconstructions, expanding surgical indications and thus reducing PN (5). This takes on even greater significance when considering that the vast majority of authors, all present included, consider the state of $\mathrm{PN}$ a disease itself, that might critically impair cardiac and respiratory function and ultimately reduce the patients' postoperative quality of life. Therefore, in recent years, the general consensus reached on this issue is that the entire lung removal should be considered only when indispensable to obtaining tumorfree resection margins $(6,7)$; indeed, the primary oncologic goal still remains a complete oncologic excision.

This article focuses on the main technical aspects of bronchial and PA reconstructive surgery with reference to the relevant results from a large number of published reports on these experiences.

\section{Preoperative assessment}

It is not always easy to identify the correct indications for carrying out a reconstructive procedure before surgery; nevertheless, careful and complete preoperative staging is 
all the more important when planning a potential bronchial or vascular reconstructive operation.

Computed tomography (CT) scans with intravenous contrast represent, in most of cases, the first-choice diagnostic tool. If bronchial infiltration is suspected, the actual presence and extent of endoscopic invasion must be assessed through a preoperative fiberoptic bronchoscopy; this examination would ideally be performed by one of the operating surgeons. After induction treatments, it should be possible to compare the endoscopic status of the bronchi to that observed before therapy, where feasible. Nevertheless, in a few patients, intraoperative exploration may show a tumor infiltration limited to the external surface of the bronchial wall, not visible during bronchoscopic evaluation; in such cases, stiffness of the bronchial wall may be an indirect endoscopic marker of peribronchial infiltration. Endobronchial ultrasonography has further improved the accuracy of fiberoptic bronchoscopy in recent years. However, establishing the correct indication for a plasty of the PA based solely on preoperative imaging assessments could turn out to be complicated. PA infiltration degree and extension are even more difficult and controversial to define after neoadjuvant therapy. At preoperative restaging CT, it is usually hard to distinguish the diffuse desmoplastic reaction and fibrosis related to chemotherapy and radiotherapy from true residual tumor. Aortography and magnetic resonance angiography (MRA) can provide useful additional information to assess the pattern of vascular invasion; however, preoperative radiological evidence may overestimate or underestimate the vascular involvement and be responsible for erroneous indications (8). Therefore, the final decision to perform a reconstructive procedure as an alternative to standard lobectomy or PN is usually made by the surgeon according to intraoperative findings.

\section{Indications and operative technique}

In patients presenting a centrally located lung neoplasm, the traditional indication for a bronchoplasty, a PA reconstruction, or both, is a tumor which extends to the origin of a lobar bronchus and/or beyond the root of a lobar branch of the PA, but without involving the residual lobes to the degree which would necessitate a PN. Sleeve resection may be also indicated in the event that the bronchus and/ or the PA are infiltrated by a metastatic N1 lymph node with extracapsular extension, as is often the case with patients affected by left upper lobe malignancies, requiring a combined bronchovascular reconstruction. Moreover, the occurrence of indissociable hilar scar tissue, as a result of a previous neoadjuvant chemotherapy regimen, can represent an additional indication for reconstructive operations.

Because the presence of fibrosis or inflammatory tissue response may pose doubts on the completeness of surgical removal of the tumor, the intraoperative pathology assessment of resection margins by frozen section analysis is mandatory in order to choose the most appropriate resection to be undertaken. Technical expertise and mature surgical judgment are needed, because it is generally in this step of the operation that the tumor can be judged amenable to a sleeve resection or a $\mathrm{PN}$, or considered unresectable.

Such operations may be readily accomplished through a thoracotomic approach. Either posterolateral or lateral muscle-sparing incision can provide the appropriate exposure of the hilum and fissures, thus allowing for an accurate dissection of the bronchial tree and proximal vessels. Nonetheless, some reports of success with different VATS sleeve resections have recently been reported by a few authors $(9,10)$.

The large majority of these reconstructive procedures are performed for tumors originating in the upper lobes, while the need for bronchovascular reconstruction in patients with a tumor of the lower and/or middle lobe is infrequent.

\section{Standard upper bronchial sleeve resections}

On the right side, dissection of the airway begins from the antero-superior aspect of the upper lobe bronchus (ULB). Next, the lung is retracted anteriorly and the bifurcation between the ULB and the intermediate bronchus is exposed. The intrafissural artery is detected through dissecting the fissure just behind the middle lobe hilum, proceeding from front to back. During this phase, it is essential to ensure that the arterial branches to the middle and the lower lobe are free from disease; otherwise, a wider parenchymal excision (even a PN) should be considered. The posterior portion of the fissure can be completed with a linear stapler once the arterial branch to the superior segment of the lower lobe is detected. This can be easily identified by continuing the dissection of the PA from behind, in the space between where the ULB takes off and the intermediate bronchus; in this way, it is possible to avoid an extensive parenchymal dissection at the fissure level, and subsequently reduce the incidence of air leaks. The intermediate bronchus is encircled just distal to the right upper lobe take-off and gently hung by umbilical tape to aid the appropriate airway transection. Once resectability is confirmed, the pulmonary 
arterial branches to the upper lobe are ligated and divided. Similarly, the pulmonary vein (PV) branch draining the upper lobe is sutured and divided, and care is taken to preserve the middle lobe venous drainage. The residual parenchymal tissue at the minor fissure is divided through a linear stapler. Next, the full control of the mainstem bronchus is achieved by encircling its origin with umbilical tape. The mainstem bronchus is transected first, just above the origin of the ULB. Thereafter, the intermediate bronchus is divided below the right upper lobe take-off. The cutting lines, so obtained, must be perpendicular to the long axis of the airway.

On the left side, proximal arterial control is achieved by taking care to prevent injury to the short apical-posterior segmental branch of the left PA. Dissection is continued along the plane of the artery until the superior segmental branch to the lower lobe is identified, thus allowing the posterior fissure to be completed with a linear stapler. Next, the arterial segmental branches to the upper lobe are ligated and divided. The upper PV is sutured and divided too, thereby exposing the ULB. The anterior portion of the fissure is completed with a linear stapler. The mainstem bronchus is encircled proximal to the bifurcation by umbilical tape and transected first. Next, the left lower lobe bronchus is divided at its origin; during this procedure, care must be taken when cutting up the airway to preserve the integrity of the superior segmental bronchus of the lower lobe which may rise quite close to the cutting line.

The final choice to undertake a sleeve lobectomy (SL) may be considered only after the airways have been divided and supported by macroscopic and microscopic findings. In the event of microscopic tumor invasion of the bronchial margins on frozen section analysis, additional resection of the involved stump or even PN may be required.

Once the objective of a complete tumor resection is achieved and microscopically confirmed, the anastomotic time is started. Over the years, different reconstruction techniques have been proposed. Even though the employment of a continuous (complete or partial) running suture has been reported by other investigators $(7,11)$, the current authors' preference is to undertake the anastomosis by placing interrupted stitches of $4 / 0$ monofilament absorbable material in an "outside-to-inside" fashion (12). In our technique, the first few stitches are placed starting from the edge between the cartilaginous and membranous bronchus on the mediastinal side and by continuing along the membranous wall; these initial sutures can be immediately tied in order to prevent bronchial torsion on its axis, and, ultimately, to confer improved stability on the anastomosis. Additional stitches are placed moving towards the lateral side to complete the first half of the cartilaginous suture. Once the midpoint of the cartilaginous arch is reached, the anastomosis is completed on the opposite side in a similar fashion. The stitches are then tied one by one, starting from either end of the cartilaginous bronchus and working toward the middle, thus gently dilating the circumference of the distal airway. These technical measures make it possible to compensate for even large-caliber discrepancies between the two bronchial ends.

\section{Less frequent bronchial sleeve resections}

The replant of the ULB following a lower left or middlelower right lobectomies (the so called "Y" sleeve resections) is infrequent. Right side sleeve bilobectomy is performed for an endoluminal lesion in the bronchus intermedius which extends upwards, close to the upper lobe orifice, which prevents performing a standard bilobectomy. The right mainstem bronchus is divided in the proximity of the right upper lobe take-off; the right ULB is incised at its origin and then anastomosed to the mainstem bronchus. When performing the anastomosis, special care must be taken to avoid torsion of the bronchial axis on account of the reorientation of the ULB after removal of the middle and lower lobes. Concerning the appreciable size discrepancy among mainstem and upper bronchial stumps, this can be successfully overcome by a careful and precise placement of the sutures, as reported in the previous paragraph.

On the left, a "Y" sleeve resection must be considered when the tumoral infiltration rises at the origin of the lower lobe bronchus, but must spare the upper lobe orifice (Figure 1). After completion of the bronchial dissection, umbilical tape is passed around the left mainstem bronchus and the left ULB; the latter is divided at its origin. Next, the mainstem bronchus is transected close to the lobar bifurcation. Care must be taken when dividing the left ULB to ensure the integrity of the lingular bronchus which may arise on the edge of the section line (Figure 2). Lastly, the anastomosis is harvested according to the description above (Figure 3). However, if the caliber discrepancy between the left mainstem and the ULB appears to be negligible, a partial running suture has to be considered, particularly on the mediastinal side due to its poor exposure (13).

It is rare that a middle lobe sleeve resection needs to be performed. Transection of the middle lobe vein first provides the proper exposure of the respective lobar 


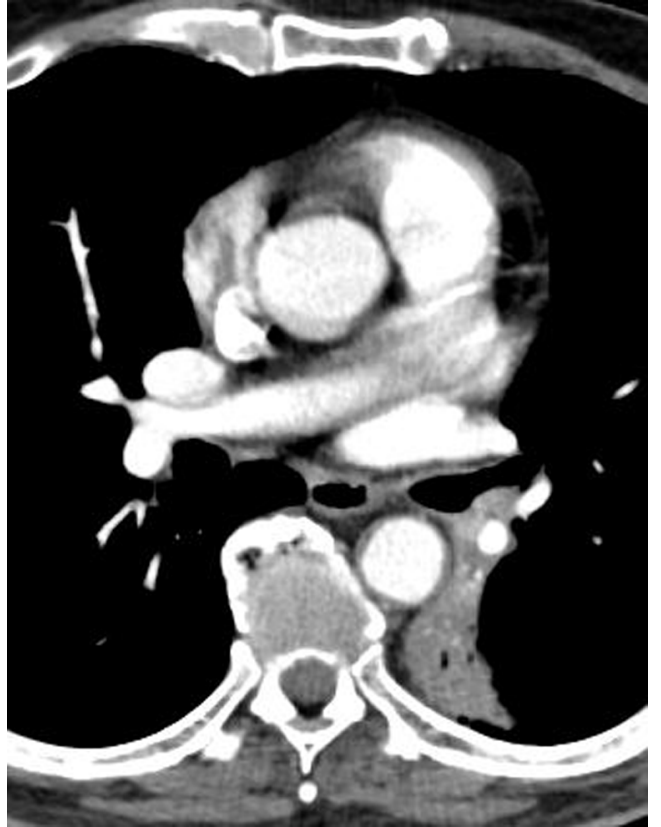

Figure $1 \mathrm{CT}$ scan image illustrating a tumor arising from the origin of the left lower bronchus. CT, computed tomography.

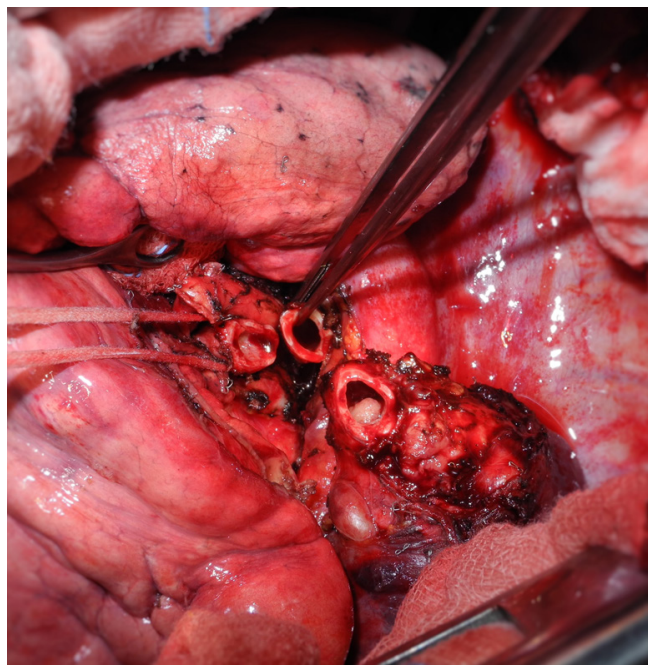

Figure 2 Intraoperative picture of a left lower sleeve lobectomy. The bronchi have been resected out and the tumor is still visible at the origin of the lower bronchus.

bronchus, which is followed to its origin. The bronchus intermedius is suspended by a right-angled clamp and divided above the origin of the middle lobe bronchus; the bronchus is then divided immediately downstream from it. The course of the proximal and distal transection lines on the bronchial ends must be mildly oblique in order to secure

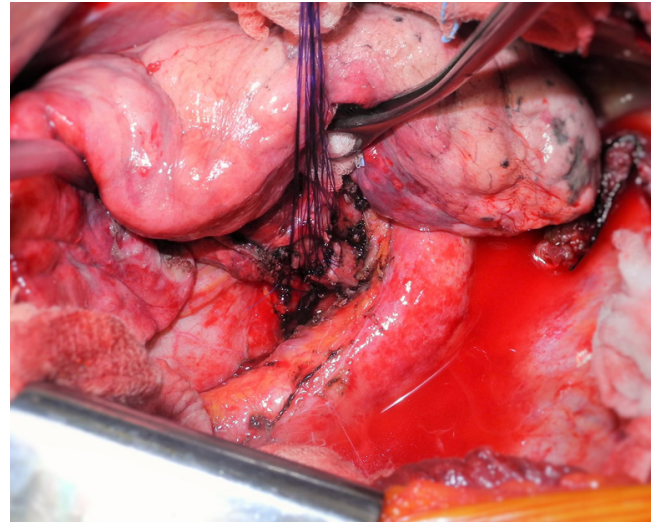

Figure 3 Intraoperative picture showing the completed anastomosis between the left mainstem bronchus and the upper bronchus after left lower sleeve lobectomy.

the orifice to the superior segment of the right lower lobe. When dividing the bronchus, care must be taken to avoid PA injury, as it lies directly posterior and slightly superior to this airway. Finally, the arterial branches to the middle lobe are ligated and sectioned. The bronchial anastomosis is performed in accordance with the previously described technique.

\section{Perioperative management of the airway}

Upon completion of the anastomotic time, the bronchial suture is checked for air leakage by the use of a pneumatic test, which entails filling the pleural cavity with a sterile hydric solution. Needle hole air leaks can be generally ignored. However, whereas a small leak through the bronchial edges may be reinforced with simple interrupted sutures, a larger area of leakage requires replacement of the entire anastomosis.

To minimize the risk of dehiscence after bronchial reconstruction, it is essential to avoid tension on the anastomotic site. This can be achieved by dividing the pulmonary ligament or, more often on the right side, by opening the pericardium around the PVs.

Protection of the bronchial anastomosis with a viable tissue flap is recommended by most authors $(14,15)$; this is even more appropriate if a double bronchoplasty and PA reconstruction have been carried out. The current authors routinely adopt an intercostal muscle flap, which provides excellent vascularization by its intercostal artery, enables preservation of airtightness even in the event of small anastomotic dehiscence, and limits the risk of PA erosion, 
particularly when an associated vascular reconstruction has been performed. The technique for intercostal muscle flap harvesting and transposition has been extensively described in previous publications $(12,14)$. Because preparation of the intercostal muscle needs to be performed before the chest is opened, it is advisable to anticipate its harvest whenever there is a chance to proceed with a bronchovascular procedure. In the absence of an available intercostal muscle flap, a mediastinal fat pad, parietal pleura, or pericardium can also be harvested and transposed (15).

The administration of systemic low-dose steroids in the immediate post-operative period is completed on a routine basis in order to obtain antiedema effect, thus reducing retention of secretions and atelectasis, improving parenchymal expansion, and minimizing granulation of the mucosa at the place of reconstruction but without enhanced risk of dehiscence. Aerosolized steroids are also part of postoperative treatment.

The anastomosis is checked endoscopically at the end of the operation and before discharge; routine fiberoptic bronchoscopies are also administered at regular intervals in line with the oncologic imaging follow-up.

\section{PA reconstruction}

The left and right main branches of the PA may be directly involved by a pulmonary hilar tumor or through the contact with a metastatic lymph node to a variable extent. Moreover, the persistence of fibrotic scar tissue, especially after induction therapy, can also require a vascular reconstructive procedure as well.

In cases of marginal infiltration limited to an aspect of the arterial wall, such as in the presence of a tumor rising from the origin of a single lobar branch of the PA, a simple tangential resection with a direct repair (running suture or mechanical stapling) is generally sufficient to achieve a complete excision. Because this technique represents just a variation of the standard ligation of the vessel, it is not further considered in this article. Larger vascular defects resulting from the removal of lesions which infiltrate up to $30 \%$ of the PA wall circumference can be successfully replaced by a patch of biological or synthetic material, thus avoiding an arterial sleeve resection. A wider involvement of the vessel requires its circumferential transection with subsequent reconstruction by an end-to-end anastomosis or through the interposition of a prosthetic conduit. However, extended infiltrations of the PA, such as in the event of a left upper lobe tumor infiltrating the concave surface of the
PA from its origin down to the anterobasal artery or, on the right side, a posterolateral infiltration from the upper division arteries to the arterial branch for the superior segment of the lower lobe, necessitates PN in order to obtain the complete resection of the neoplasm.

The first step of the operation is to secure full control of the proximal PA, as detailed above. If, however, performing an upper lobectomy transection of superior PV should be postponed until the feasibility of the procedure has been determined-as is often the case-division of this vessel can facilitate exposure of the PA. Dissection in the interlobar fissure can be made safer after proximal arterial clamping. We prefer to engage the fissure plan starting from the back on the left side, at the level of the main PA, and moving on and downwards with the dissection via a subadventitial plane. On the right side, we approach the intrafissural portion of the PA by dissecting front to back, behind the middle lobe, in order to identify the branch for the superior segment of the lower lobe. To avoid extensive parenchymal dissection in the fissure, the exposure of this vessel can be continued posteriorly at the bifurcation between the ULB and the bronchus intermedius. The resection time begins once the main and distal branches of the PA, both PVs, and the bronchial divisions of interest, have been properly prepared. Over time, as technical experience has grown and mean clamping time has decreased, the anticoagulant administration has been progressively reduced to approximately 25 units $/ \mathrm{kg}$ instead of a dose between 3,000 and 5,000 units as was used in the past (16). Nowadays, we normally administer 1,500 to 2,000 IU of heparin sodium before clamping of the proximal PA. This dosage has proven to be effective in reducing postoperative oozing, especially from the lymphadenectomy sites, without an increased incidence of thrombotic events. To obtain backflow control, we now prefer to clamp the arterial vessel farther downstream from the infiltrated site than in the past when we used to clamp the inferior PV. Once the vascular reconstruction is complete, heparin is not reversed by protamine sulphate after declamping.

\section{Partial resection of the $P A$}

The traditional indication for choosing a patch plasty is a PA infiltration restricted to less than one-half of the entire circumference of the vascular wall (Figure 4); this circumstance includes a variety of conditions ranging from limited involvement of the origin of a segmental artery to larger defects resulting from the longitudinal excision of an 


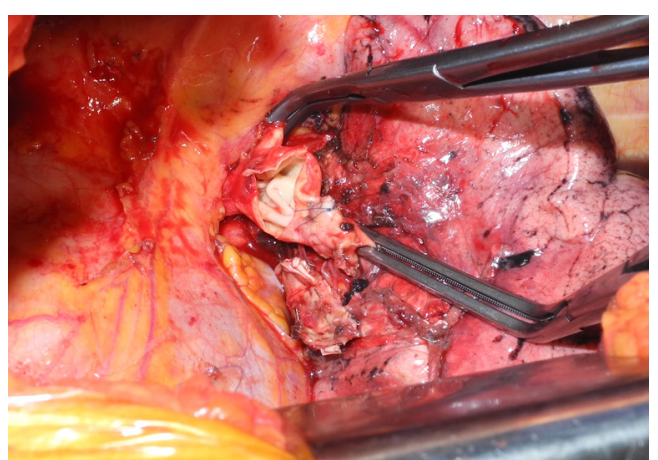

Figure 4 Intraoperative picture during a patch reconstruction of the PA. The tumor infiltrating the left PA has been resected; the $\mathrm{PA}$ is under cross-clamping and the defect is visible. PA, pulmonary artery.

invasive malignancy. In some cases of longitudinal extended defects (more than $2.5 \mathrm{~cm}$ in length) on one aspect of the $\mathrm{PA}$, when the opposite side of the circumference of the vessel is free from tumor, the reconstruction by a wide patch can be considered (17). If the infiltration is more extensive, the vascular sleeve resection with subsequent end-to-end anastomosis or conduit interposition is required.

Over the years, a variety of different materials have been proposed for patch harvesting, including synthetic and biological (autologous and heterologous) options. Among the biological alternatives, pericardial tissue is widely employed and represents the authors' first choice, particularly the autologous version. The latter confers a set of advantages, such as proper thickness and mechanical resistance, wide availability on both sides of the chest that allows the repair of even large defects, and total biocompatibility compared to the heterologous materials; not of least importance, it is also cost-free. However, fresh autologous pericardium also has some technical limits because of its tendency to shrink and curl making it more difficult to tailor and stitch up the patch to the vascular wall. In contrast, the lower flexibility of the bovine pericardium and its more consistent and stiff edges facilitate reconstructive procedure. In order to improve technical features of the autologous pericardium, the present authors have developed an intraoperative method to stabilize this tissue by a glutaraldehyde-buffered solution; this fixation technique has been reported in our previous publication (18). Through this trick, it is possible to minimize the tendency of the fresh pericardial patch to retract and curl, thus allowing an easier vascular reconstruction and reducing the risk of postoperative bleeding from the suture related to the recoil of the margins. If the autologous option is chosen one, the desirable tissue can be obtained during the same operation by carving the pericardium anteriorly to the phrenic nerve, leaving open the pericardial defect. The venous patches (azygos, saphenous and superior PV) represent another viable alternative. The azygos vein wall displays sufficient mechanical characteristics, but the sampling is available only in the case of right side procedures, and it generally yields a limited amount of tissue. Conversely, a superior PV patch can typically be used during a left upper lobectomy operation, since anatomical reasons make its availability uncommon on the right side. When the extraparenchymal portion of $\mathrm{PA}$ is free from tumor, it is possible to isolate up to $2 \mathrm{~cm}$ of superior vein conduit by ligating the PV branches and then applying a thoraco-abdominal (TA) linear vascular stapler on the proximal portion of the vein, next to the pericardial reflection. After venous transection, a patch can be easily obtained by trimming the PV conduit. The superior PV exhibits similar properties to the PA tissue and represents therefore a suitable reconstruction material. This is not equally true about the saphenous vein which requires a separate procedure to be harvested and has valves on its endothelial surface.

Once the patch has been collected and properly tailored, it is secured to the arterial wall by two stay stiches applied to the opposite apices of the vascular defect. The suture (5-0 or 6-0 monofilament non-absorbable material) is carried out in a running fashion with a top-down approach and then proceeding back to the top, while the assistant grasps and stretches the patch (Figure 5). The inferior stay suture is not tied: it is used only to keep the patch in place until the suture line has reached its level. In patients who will have to undergo a combined bronchial reconstruction, the PA patch anastomosis is generally performed first in order to reduce clamping time.

After residual lung re-expansion, haemostasis at the level of the vascular suture must be checked again since variation of the tensile forces on the PA surface may result in bleeding due to the retraction of the patch margins, especially when the fresh autologous pericardium has been used.

\section{Sleeve resection of the $P A$}

When a tumor involves one half or more of the whole circumference of the vessel, the PA sleeve resection is required. In this event, caliber discrepancy and unexpected traction between the vascular stumps represent the major technical issues. When dividing the artery, it is advisable to 


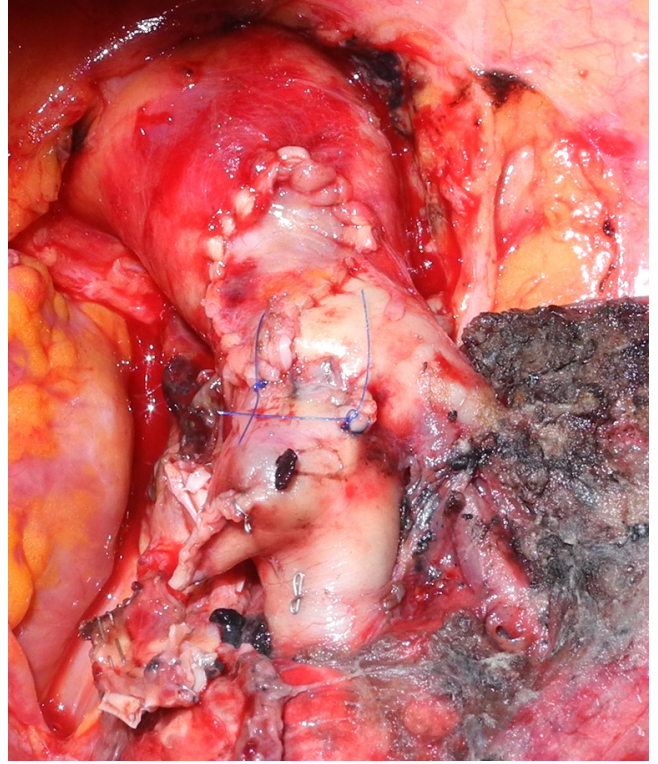

Figure 5 Intraoperative picture during a patch reconstruction of the PA. The patch reconstruction of the left PA is complete. PA, pulmonary artery.

obtain regular transection edges and a uniform endothelial surface on both the vascular stumps, even at the cost of some lost tissue. This helps the accurate placement of the stitches in order to gain a uniform suture. Homogeneous margins can also facilitate the correction of large caliber discrepancy, if it occurs. The anastomosis is performed by 5-0 or 6-0 running sutures in monofilament nonabsorbable material; these must be applied very carefully to avoid postoperative stenosis. For the ultimate success of the reconstructive procedure, it is essential to achieve a tenseness-free anastomotic site. Tension release can be improved by sectioning the inferior pulmonary ligament or, on the right side, by opening the pericardium around the inferior PV.

In those patients undergoing simultaneous reconstructive procedure on the airway, the bronchial sleeve resection leads to a shortening of the bronchial axis, thus achieving an easier approximation of the vascular stumps with usual acceptable tension. The end-to-end PA suture is performed last thereby minimizing the manipulation of the reconstructed vessel. Indeed, on completion of the bronchial anastomosis, the distance between the two arterial ends is markedly reduced, and it can be further decreased by elevating the lower lobe while suturing. Restoration of the blood flow and removal of the proximal clamp generally relieve any residual tension. However, if the distance between the two arterial stumps is still deemed significant despite these measures, the interposition of a prosthetic conduit should be considered.

At times, the isolated sleeve resection of an extended segment of the main PA may be required. This usually results in excessive distance between the two vascular stumps with subsequent high tension on the anastomotic site. Such technical situations may occur, typically on the left side in those cases requiring the removal of a long portion of the PA without associated bronchial sleeve resection since the lobar bronchus is free from tumor infiltration. In these sporadic cases, the vascular reconstruction cannot be performed by a direct end-to-end suture and a prosthetic conduit interposition is needed.

Although these procedures are infrequent, over the years several materials and techniques for tailoring and replacement of the vascular conduit have been proposed. Biological materials represent the preferred choice because of their higher biocompatibility and the lower risk of postoperative thrombosis. On this issue, the authors have previously reported the use of autologous pericardium with satisfactory results $(19,20)$. More recently, we have also implemented the heterologous pericardium (bovine and porcine) as an alternative (17). When the autologous pericardium is employed, the epicardial surface is oriented inside the lumen of the conduit. According to the current authors' original intraoperative procedure, the pericardial leaflet is trimmed to a rectangular shape, wrapped around a chest tube or a syringe of appropriate diameter, and sutured longitudinally. In our initial experience, this suture was performed manually with a 6-0 monofilament nonabsorbable material. More recently, we have proposed a technical alternative which includes the use of a mechanical linear stapler (vascular load) to suture the longitudinal aspect of the pericardial conduit.

An interesting, recently introduced option for the arterial conduit replacement is the extraparenchymal portion of the superior PV $(21,22)$. This can be harvested from the resected upper lobe when the vessel is not directly involved. The technique to obtain an up to $20-\mathrm{mm}$ PV conduit has been fully described above. The venous conduit is an ideal substitute for a PA replacement since it has adequate thickness and structural similarity with the arterial wall. It is advisable to tailor the length of the biological conduit on the basis of the resected arterial segment, because the elasticity of the two tissues is comparable.

During replacement, the running proximal suture (monofilament material) is completed first; the distal 
suture is then completed with the same technique once the conduit length has been established. Care must be taken to avoid making the conduit too long, which may cause kinking of the vessel, impair blood flow, and therefore cause thrombosis.

\section{Final considerations}

Bronchoplasty and pulmonary arterioplasty procedures combined with lobectomy, although technically demanding with respect to standard major lung resections, have provided excellent results, comparable or even better than those obtained by $\mathrm{PN}$ (1-4,6,7,23-30).

The current results from the literature support the increasingly widespread use of bronchovascular reconstructive operations for the treatment of centrally located lung tumors, even in functionally normal patients. These surgical procedures actually provide the complete oncologic resection of $\mathrm{PN}$ with the functional advantages of standard lobectomy, including when performed after induction chemotherapy. When planning such operations, the thorough preoperative assessment of tumor extent, a careful surgical technique, and vigilant perioperative management are mandatory in order to achieve the best possible outcome for the patient with the least cost.

\section{Acknowledgments}

Funding: None.

\section{Footnote}

Conflicts of Interest: The authors have no conflicts of interest to declare.

Ethical Statement: The authors are accountable for all aspects of the work in ensuring that questions related to the accuracy or integrity of any part of the work are appropriately investigated and resolved.

Open Access Statement: This is an Open Access article distributed in accordance with the Creative Commons Attribution-NonCommercial-NoDerivs 4.0 International License (CC BY-NC-ND 4.0), which permits the noncommercial replication and distribution of the article with the strict proviso that no changes or edits are made and the original work is properly cited (including links to both the formal publication through the relevant DOI and the license).
See: https://creativecommons.org/licenses/by-nc-nd/4.0/.

\section{References}

1. Park JS, Yang HC, Kim HK, et al. Sleeve lobectomy as an alternative procedure to pneumonectomy for non-small cell lung cancer. J Thorac Oncol 2010;5:517-20.

2. Shi $W$, Zhang $W$, Sun $H$, et al. Sleeve lobectomy versus pneumonetomy for non -small cell lung cancer. World J Surg Oncol 2012;10:265.

3. Maurizi G, D'Andrilli A, Anile M, et al. Sleeve lobectomy compared with pneumonectomy after induction therapy for non-small lung cancer. J Thorac Oncol 2013;8:637-43.

4. Nagayasu T, Yamasaki N, Tsuchiya T, et al. The evolution of bronchoplasty and broncho-angioplasty as treatments for lung cancer. Evolution of 30 years of data from a single institution. Eur J Cardiothorac Surg 2016;49:300-6.

5. Maurizi G, Rendina EA. Bronchovascular reconstructions for lung cancer: improvements over time. Eur J Cardiothorac Surg 2016;49:306-7.

6. Takeda S, Maeda H, Koma M, et al. Comparison of surgical results after pneumonectomy and sleeve lobectomy for non-small cell lung cancer: trends over time and 20-year institutional experience. Eur J Cardiothorac Surg 2006;29:276-80.

7. Gómez-Caro A, Garcia S, Reguart N, et al. Determining the appropriate sleeve lobectomy versus pneumonectomy ratio in central non-small cell lung cancer patients: an audit of an aggressive policy of pneumonectomy avoidance. Eur J Cardiothorac Surg 2011;39:352-9.

8. Ciccone AM, D'Andrilli A, Venuta F, et al. Imaging of tumor infiltration of the pulmonary artery amenable to sleeve resection. J Thorac Cardiovasc Surg 2008;136:229-30.

9. Mahtabifard A, Fuller CB, McKenna RJ Jr. Vido-assisted thoracic surgery sleeve lobectomy: a case series Ann Thorac Surg 2008;85:S729-32.

10. Gonzalez-Rivas D, Fernandez R, Fieira E, et al. Uniportal video-assisted thoracoscopic bronchial sleeve lobectomy: first report. J Thorac Cardiovasc Surg 2013;145:1676-7.

11. Fadel E, Yldizeli B, Chapelier A, et al. Sleeve lobectomy for bronchogenic cancers: factors affecting survival. Ann Thorac Surg 2002;74:851-8.

12. Rendina EA, Venta F, Ciriaco P, et al. Bronchovascular sleeve resection. Technique, perioperative management, prevention and treatment of complications. J Thorac Cardiovasc Surg 1993;106:73-9.

13. Maurizi G, Ciccone AM, Vanni C, et al. Reimplantation of 
the upper lobe bronchus after lower sleeve lobectomy or bilobectomy: long-term results. Eur J Cardiothorac Surg 2018;53:1180-5.

14. Rendina EA, Venuta F, Ricci P, et al. Protection and revascularization of bronchial anastomoses by the intercostal pedicle flap. J Thorac Cardiovasc Surg 1994;107:1251-4.

15. Tsuchiya R. Bronchoplastic techniques. In: Patterson GA, Deslauriers J, Lerut A, et al. editors. Pearson's Thoracic and Esophageal Surgery 2nd ed. Philadelphia, PA: Churchill Livingstone, 2002:1005.

16. Ricci C, Rendina EA, Venuta F, et al. Reconstruction of the pulmonary artery in patients with lung cancer. Ann Thorac Surg 1994;57:627.

17. D'Andrilli A, Maurizi G, Ciccone AM, et al. Long-segment pulmonary artery resection to avoid pneumonectomy: long-term results after prosthetic replacement. Eur J Cardiothorac Surg 2018;53:331-5.

18. D'Andrilli A, Ibrahim M, Venuta F, et al. Glutaraldhehyde preserved autologous pericardium for patch reconstruction of the pulmonary artery and superior vena cava. Ann Thorac Surg 2005;80:357-8.

19. Rendina EA, Venuta F, De Giacomo T, et al. Reconstruction of the pulmonary artery by a conduit of autologous pericardium. J Thorac Cardiovasc Surg 1995;110:867-8.

20. Venuta F, Ciccone AM, Anile M, et al. Reconstruction of the pulmonary artery for lung cancer: long-term results. J Thorac Cardiovasc Surg 2009;138:1185-91.

21. Cerezo F, Cano JR, Espinosa D, et al. New technique for pulmonary artery reconstruction. Eur J Cardiothorac Surg 2009;36:422-3.

22. D'Andrilli A, Maurizi G, Andreetti C, et al. Pulmonary artery reconstruction with pulmonary vein conduit for lung cancer: medium-term results. Ann Thorac Surg 2014;98:990-5.

doi: $10.21037 /$ ccts.2019.05.01

Cite this article as: Maurizi G, Vanni C, D'Andrilli A, Ciccone AM, Ibrahim M, Andreetti C, Menna C, Poggi C, Venuta F, Rendina EA. Bronchoplasty and pulmonary arterioplasty. Curr Chall Thorac Surg 2019;1:3.
23. Okada M, Yamagashi H, Stak S, et al. Survival related to lymph node involvement in lung cancer after sleeve lobectomy compared with pneumonectomy. J Thorac Cardiovasc Surg 2000;119:814-9.

24. Deslauriers J, Grégoire J, Jacques LF, et al. Sleeve lobectomy versus pneumonectomy for lung cancer: a comparative analysis of survival and sites of recurrence. Ann Thorac Surg 2004;77:1152-6.

25. Ludwig C, Stoelben E, Olschewski M, et al. Comparison of morbidity, 30-day mortality, and long term survival after pneumonectomy and sleeve lobectomy for non-small cell lung carcinoma. Ann Thorac Surg 2005;79:968-73.

26. Bagan P, Berna P, Pereira JC, et al. Sleeve lobectomy versus pneumonectomy: tumor characteristics and comparative analysis of feasibility and results. Ann Thorac Surg 2005;80:2046-50.

27. Kim Y T, Kang CH, Sung SW, et al. Local control of disease related to lymph node involvement in non-small cell lung cancer after sleeve lobectomy compared with pneumonectomy. Ann Thorac Surg 2005;79:1153-61.

28. Melloul E, Egger B, Krueger T, et al. Mortality, complications and loss of pulmonary function after pneumonectomy vs. sleeve lobectomy in patients younger and older than 70 years. Interact Cardiovasc Thorac Surg 2008;7:986-9.

29. Ma Z, Dong J, Fan J, et al. Does sleeve lobectomy concomitant with or without pulmonary artery reconstruction (double sleeve) have favorable results for non-small cell lung cancer compared with pneumonectomy? A meta-analysis. Eur J Cardiothorac Surg 2007;32:20-8.

30. D'Andrilli A, Maurizi G, Andreetti C, et al. Sleeve Lobectomy Versus Standard Lobectomy for Lung Cancer: Functional and Oncologic Evaluation. Ann Thorac Surg 2016;101:1936-42. 